\title{
Revising Z: Part II - logical development
}

\author{
Martin C. Henson ${ }^{1}$ and Steve Reeves ${ }^{2}$ \\ ${ }^{1}$ Department of Computer Science, University of Essex, UK; \\ ${ }^{2}$ Department of Computer Science, University of Waikato, New Zealand
}

Keywords: Specification language Z; Logics of specification languages; Semantics of specification languages

\begin{abstract}
This is the second of two related papers. In "Revising Z: Part I logic and semantics" (this journal) we introduced a simple specification logic $\mathrm{Z}_{C}$ comprising a logic and a semantics (in ZF set theory). We then provided an interpretation for (a rational reconstruction of) the specification language $\mathrm{Z}$ within $Z_{C}$. As a result we obtained a sound $\operatorname{logic}$ for $Z$, including the basic schema calculus. In this paper we extend the basic framework with more sophisticated features (including schema operations) and we mount a critique of a number of concepts used in Z. We further demonstrate that the complications and confusions which these concepts introduce can be avoided without compromising expressibility.
\end{abstract}

\section{Introduction}

In the earlier companion paper [HeR99] we introduced a specification system $\mathrm{Z}_{C}$, a typed set theory incorporating the notion of a schema type and we established a number of meta-mathematical results including its soundness with respect to a simple set-theoretic semantics. We went on to introduce a notation very much closer to the $\mathrm{Z}$ familiar in the literature. This included more substantial mechanisms for the construction of propositions, sets and, in particular, schemas.

In this paper we further extend the logic for Z substantially. In Section 2, we extend the schema calculus with a number of new forms of expression and introduce a number of other new forms of term, set and propositional forms. We then turn, in Section 3, to consider two $\mathrm{Z}$ concepts, $\theta$ expressions and schema priming, which are without doubt the locus of much confusion in the literature. 
After reviewing these notions we go on to provide additional mechanisms suitable to either interpret or modify them. Finally, in Section 4, we examine some example specifications from the literature which employ the full range of the apparatus available in $\mathrm{Z}$ and demonstrate how such specifications may be rendered in our framework.

We do not review the logical development of [HeR99] in this paper, and the reader will need to refer to that in order to establish the logical systems which are the point of departure here.

\section{Derived constructs}

We indicated in [HeR99] that, given the basic connectives and set (hence schema) operations we introduced, we would be able to construct others that we expect to find in Z. Since we are particularly concerned with developing the logic of $\mathrm{Z}$, it is appropriate that we go on to examine the expected logical consequences of these constructions. We shall, in particular, spend some time extending the schema calculus which is of major importance in Z. In fact it is not quite clear why this has traditionally been referred to as a schema calculus since the literature typically introduces nothing beyond a notation for schema expressions. It is true that there are some suggested rules for membership in compound schemas, at least for the simplest cases like conjunction, in the normative source [Nic95], but these are quite clearly wrong. For example (ibid. Section F.6.6, p. 207) we can easily derive the following rules, the first of which is too restrictive and the others are not even well-formed:

$$
\frac{\Gamma \vdash b \in S \quad \Gamma \vdash b \in T}{\Gamma \vdash b \in S \wedge T} \quad \frac{\Gamma \vdash b \in S \wedge T}{\Gamma \vdash b \in S} \quad \frac{\Gamma \vdash b \in S \wedge T}{\Gamma \vdash b \in T}
$$

using the rules (SchBindMem) and $(S A n d)$. We are not the only authors to detect this mistake in [Nic95]. However, the suggested remedy (adding a proviso to rule (SchBindMem) [Mar98]) only ensures that the elimination rules above are well-defined: they are still very limited. Beyond this incorrect treatment of the simplest aspects of the schema calculus there is almost nothing, for example the incomplete rule for schema composition (SComp) (ibid. p. 208). These errors were carried over into [BrM96] but are rectified in [Mar98].

\subsection{Schema conjunction}

We would expect to define conjunction over schemas by analogy with operations like set intersection and logical conjunction:

$$
\llbracket S_{0} \wedge S_{1} \rrbracket=_{d f} \llbracket \neg\left(\neg S_{0} \vee \neg S_{1}\right) \rrbracket
$$

Using rules $\left(Z_{\neg S}\right)$ and $\left(Z_{\vee S}\right)^{1}$ we obtain the following derived rule for type assignment:

$$
\frac{\Gamma \triangleright S_{0}: \mathbb{P} T_{0} \quad \Gamma \triangleright S_{1}: \mathbb{P} T_{1}}{\Gamma \triangleright S_{0} \wedge S_{1}: \mathbb{P}\left(T_{0} \vee T_{1}\right)}\left(Z_{\wedge S}\right)
$$

${ }^{1}$ A reminder: these rules and many others that follow are from [HeR99]. 
We can simplify the right-hand side of the definition, so that we have, for fresh $y$ :

$$
\llbracket S_{0}^{\mathbb{P} T_{0}} \wedge S_{1}^{\mathbb{P} T_{1}} \rrbracket=\left\{y: T_{0} \vee T_{1} \mid y \uparrow T_{0} \in \llbracket S_{0} \rrbracket \wedge y \uparrow T_{1} \in \llbracket S_{1} \rrbracket\right\}
$$

The following introduction rule is derivable: $\left(\wedge^{+}\right)$and $\left(\{\}^{+}\right)$:

$$
\frac{\Gamma \vdash t \uparrow T_{0} \in S_{0} \quad \Gamma \vdash t \uparrow T_{1} \in S_{1} \quad \Gamma^{-} \triangleright t: T_{0} \vee T_{1}}{\Gamma \vdash t \in S_{0} \wedge S_{1}}\left(S_{\wedge}^{+}\right)
$$

The corresponding elimination rules:

$$
\begin{aligned}
& \frac{\Gamma \vdash t \in S_{0} \wedge S_{1} \quad \Gamma^{-} \triangleright S_{0}: \mathbb{P} T_{0}}{\Gamma \vdash t \uparrow T_{0} \in S_{0}}\left(S_{\wedge_{\circ}}^{-}\right) \\
& \frac{\Gamma \vdash t \in S_{0} \wedge S_{1} \quad \Gamma^{-} \triangleright S_{1}: \mathbb{P} T_{1}}{\Gamma \vdash t \uparrow T_{1} \in S_{1}}\left(S_{\wedge_{\mathrm{I}}}^{-}\right)
\end{aligned}
$$

With these in place we can prove the expected relationship (see [WoD96] pp. 165-6):

\section{Lemma 2.1.}

$$
\frac{\Gamma^{-} \triangleright\left[D_{0}^{*} \mid P_{0}\right]: \mathbb{P} T_{0} \quad \Gamma^{-} \triangleright\left[D_{1}^{*} \mid P_{1}\right]: \mathbb{P} T_{1}}{\Gamma \vdash\left[D_{0}^{*} \mid P_{0}\right] \wedge\left[D_{1}^{*} \mid P_{1}\right]=\left[D_{0}^{*} \vee D_{1}^{*} \mid P_{0} \wedge P_{1}\right]}\left(\wedge^{=}\right)
$$

Proof. We can use the equational logic that we already have at our disposal. The result follows easily by rules $\left(\neg^{=}\right)$and $\left(\mathrm{V}^{=}\right)$and De Morgan's laws.

Finally we have the substitution rule:

$$
\frac{S_{0}=S_{2} \quad S_{1}=S_{2}}{S_{0} \wedge S_{1}=S_{2} \wedge S_{3}}
$$

Again, these follow easily from the corresponding rules for disjunction and negation schemas.

\subsection{Schema implication}

Following the pattern given above for conjunction, we would expect the definition:

$$
\llbracket S_{0} \Rightarrow S_{1} \rrbracket=_{d f} \llbracket \neg S_{0} \vee S_{1} \rrbracket
$$

Using the rules $\left(Z_{\neg S}\right)$ and $\left(Z_{\vee S}\right)$ we obtain a derived rule for type assignment:

$$
\frac{\Gamma \triangleright S_{0}: \mathbb{P} T_{0} \quad \Gamma \triangleright S_{1}: \mathbb{P} T_{1}}{\Gamma \triangleright S_{0} \Rightarrow S_{1}: \mathbb{P}\left(T_{0} \vee T_{1}\right)}\left(Z_{\Rightarrow S}\right)
$$

Simplifying the right-hand side of the definition, we obtain, for fresh $z$ :

$$
\llbracket S_{0}^{T_{0}} \Rightarrow S_{1}^{T_{1}} \rrbracket=\left\{z: T_{0} \vee T_{1} \mid z \uparrow T_{0} \notin \llbracket S_{0} \rrbracket \vee z \uparrow T_{1} \in \llbracket S_{1} \rrbracket\right\}
$$

The introduction rule is:

$$
\frac{\Gamma, t \uparrow T_{0} \in S_{0} \vdash t \uparrow T_{1} \in S_{1} \quad \Gamma^{-} \triangleright t: T_{0} \vee T_{1} \quad \Gamma^{-} \triangleright S_{0}: \mathbb{P} T_{0}}{\Gamma \vdash t \in S_{0} \Rightarrow S_{1}}\left(S_{\Rightarrow}^{+}\right)
$$


The elimination rule is:

$$
\frac{\Gamma \vdash t \in S_{0} \Rightarrow S_{1} \quad \Gamma \vdash t \uparrow T_{0} \in S_{0} \quad \Gamma^{-} \triangleright S_{1}: \mathbb{P} T_{1}}{\Gamma \vdash t \uparrow T_{1} \in S_{1}}\left(S_{\Rightarrow}^{-}\right)
$$

The expected relationship holds:

\section{Lemma 2.2.}

$$
\frac{\Gamma^{-} \triangleright\left[D_{0}^{*} \mid P_{0}\right]: \mathbb{P} T_{0} \quad \Gamma^{-} \triangleright\left[D_{1}^{*} \mid P_{1}\right]: \mathbb{P} T_{1}}{\Gamma \vdash\left[D_{0}^{*} \mid P_{0}\right] \Rightarrow\left[D_{1}^{*} \mid P_{1}\right]=\left[D_{0}^{*} \vee D_{1}^{*} \mid P_{0} \Rightarrow P_{1}\right]}
$$

Proof. Using the equational logic: rules $\left(\neg^{=}\right)$and $\left(\mathrm{V}^{=}\right)$.

Finally we have the expected substitution rule:

$$
\frac{S_{0}=S_{1} \quad S_{2}=S_{3}}{S_{0} \Rightarrow S_{2}=S_{1} \Rightarrow S_{3}}
$$

\subsection{Schema inclusion}

Schema inclusion can be defined in terms of schema conjunction.

$$
\llbracket\left[D_{0} ;\left[D_{1} \mid P_{1}\right] \mid P_{0}\right] \rrbracket={ }_{d f} \llbracket\left[D_{0} \vee D_{1} \mid P_{0}\right] \wedge\left[D_{1} \mid P_{1}\right] \rrbracket
$$

It should be noted that this, unlike the other operators we consider, is a noncompositional definition which involves a generalisation, on the left-hand side, of the language of declarations to include schema references.

The rules are then easily calculated as special cases of those for schema conjunction. First the typing rules:

$$
\frac{\Gamma \triangleright\left[D_{0} \vee D_{1} \mid P_{0}\right]: \mathbb{P}\left(T_{0} \vee T_{1}\right) \quad \Gamma \triangleright\left[D_{1} \mid P_{1}\right]: \mathbb{P} T_{1}}{\Gamma \triangleright\left[D_{0} ;\left[D_{1} \mid P_{1}\right] \mid P_{1}\right]: \mathbb{P}\left(T_{0} \vee T_{1}\right)}\left(Z_{\text {inc }}\right)
$$

The introduction rule is:

$$
\frac{\Gamma \vdash t \in\left[D_{0} \vee D_{1} \mid P_{0}\right] \quad \Gamma \vdash t \uparrow T_{1} \in\left[D_{1} \mid P_{1}\right]}{\Gamma \vdash t \in\left[D_{0} ;\left[D_{1} \mid P_{1}\right] \mid P_{0}\right]}
$$

The elimination rules are:

$$
\begin{aligned}
& \frac{\Gamma \vdash t \in\left[D_{0} ;\left[D_{1} \mid P_{1}\right] \mid P_{0}\right]}{\Gamma \vdash t \in\left[D_{0} \vee D_{1} \mid P_{0}\right]} \\
& \frac{\Gamma^{-} \triangleright\left[D_{1} \mid P_{1}\right]: \mathbb{P} T_{1} \quad \Gamma \vdash t \in\left[D_{0} ;\left[D_{1} \mid P_{1}\right] \mid P_{0}\right]}{\Gamma \vdash t \uparrow T_{1} \in\left[D_{1} \mid P_{1}\right]}
\end{aligned}
$$

Finally, we have the expected equational law:

$$
\frac{\Gamma^{-} \triangleright\left[D_{0}^{*} \mid P_{0}\right]: \mathbb{P} T_{0} \quad \Gamma^{-} \triangleright\left[D_{1}^{*} \mid P_{1}\right]: \mathbb{P} T_{1}}{\Gamma \vdash\left[D_{0}^{*} ;\left[D_{1}^{*} \mid P_{1}\right] \mid P_{0}\right]=\left[D_{0}^{*} \vee D_{1}^{*} \mid P_{0} \wedge P_{1}\right]} \quad\left(i n c^{=}\right)
$$

Although the $\Delta$ and $\Xi$ schemas of $Z$ are intimately linked, in the standard accounts, with schema inclusion, we shall delay their consideration until we discuss schema priming and the $\theta$ operator (Section 3 below). 


\subsection{Schema composition and piping}

We proceed by adopting a standard definition of schema composition, in terms of renaming, conjunction and hiding (see e.g. [Dil94]). We give a very simplified, introductory account, restricting the composition along a single pair of complementary labels.

We shall need to index the composition operator with the pair of labels along which the composition is taken. Let $S_{0}: \mathbb{P}\left(T_{0} \vee\left[l^{\prime}: T\right]\right)$ and $S_{1}: \mathbb{P}\left(T_{1} \vee[l: T]\right)$. Let $v$ be a fresh label. Then:

$$
\llbracket S_{0}{\stackrel{9}{9}\left(l^{\prime}, l\right)}_{1} S_{1} \rrbracket=_{d f} \llbracket\left(S_{0}\left[l^{\prime} \leftarrow v\right] \wedge S_{1}[l \leftarrow v]\right) \backslash[v: T] \rrbracket
$$

For notational simplicity it is sensible to define a derived operator on types:

$$
T_{0}{\stackrel{\mathrm{o}}{\left(l^{\prime}, l\right)}}_{1} T_{1}=_{d f}\left(T_{0}\left[l^{\prime} \leftarrow v\right] \vee T_{1}[l \leftarrow v]\right) \backslash[v: T]
$$

First we have the typing rule, which is easily derived:

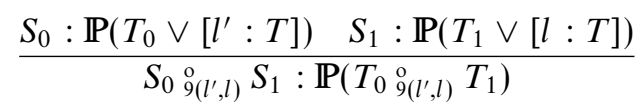

In the introduction rule we write $V_{0}$ and $V_{1}$ for $T_{0} \vee\left[l^{\prime}: T\right]$ and $T_{1} \vee$ $[l: T]$ respectively.

$$
\frac{\Gamma \vdash\left(t \uparrow V_{0}\left[l^{\prime} \leftarrow v\right]\right)\left[v \leftarrow l^{\prime}\right] \in S_{0} \quad \Gamma \vdash\left(t \uparrow V_{1}[l \leftarrow v]\right)[v \leftarrow l] \in S_{1}}{\Gamma \vdash t \uparrow\left(T_{0}^{o} \underset{g\left(l^{\prime}, l\right)}{o} T_{1}\right) \in S_{0}^{\mathbb{P} V_{0}}{ }_{9\left(l^{\prime}, l\right)}^{o} S_{1}^{\mathbb{P} V_{1}}}\left(S_{9}^{+}\right)
$$

This is calculated using the rules $\left(S_{h}^{+}\right),\left(S_{\wedge}^{+}\right)$and $\left(S_{\leftarrow}^{+}\right)$twice.

We also obtain an elimination rule:

$$
\frac{\Gamma \vdash t \in S_{0}^{\mathbb{P}\left(T_{0} \vee\left[l^{\prime}: T\right]\right)_{9}}{ }_{9\left(l^{\prime}, l\right)} S_{1}^{\mathbb{P}\left(T_{1} \vee[l: T]\right)} \quad \Gamma^{\prime} \vdash P}{\Gamma \vdash P}\left(S_{9}^{-}\right) \text {, for fresh } y_{0} \text { and } y_{1}
$$

where $\Gamma^{\prime}$ is:

$\Gamma^{-}, y_{0}: T_{0} \vee[v: T], y_{1}: T_{1} \vee[v: T] ; \Gamma^{+}, y_{0}\left[v \leftarrow l^{\prime}\right] \in S_{0}, y_{1}[v \leftarrow l] \in$ $S_{1}, y_{0} \uparrow T_{0}=t \uparrow T_{0}, y_{1} \uparrow T_{1}=t \uparrow T_{1}$.

The substitution rule is as expected:

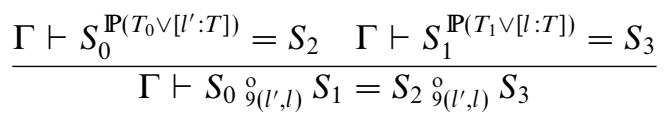

Turning now to piping, we immediately see that the definition, and therefore the rules, are much the same. All we require is to select our complementary labels to be distinguished by the diacritical marks which indicate input and output. For example, the composition operator we have introduced above, indexed by a pair of labels $(l !, l$ ?) implements the piping operator over a single input/output channel.

This section only begins an investigation of composition and piping and we have obtained rules which are rather unpleasant. Possibly the logic offers other methods for defining composition which would result in more manageable rules. Investigation of that is left to future work. 


\subsection{Schema restriction}

In view of our filtering operation on terms which we have extended to sets, we can give a pleasant definition:

$$
\llbracket S_{0}^{\mathbb{P} T_{0}} \uparrow S_{1}^{\mathbb{P} T_{1}} \rrbracket==_{d f} \llbracket S_{0} \uparrow T_{1} \wedge S_{1} \rrbracket
$$

when $T_{1} \preceq T_{0}$. When the schema $S_{1}$ is just a schema set we get:

$$
S \uparrow\left[D^{*}\right]=S \uparrow[D]
$$

This equation explains our use in this paper of the restriction symbol for term filtering. From filtered terms we were able to introduce filtered sets, and from those we obtain restricted schemas here. The overloading of the symbol is quite unambiguous: restriction involves a schema set, whereas filtering employs a schema type.

Our definition is a little less general than that in [Spi92] (p. 34) which has:

$$
\llbracket S_{0}^{\mathbb{P} T_{0}} \uparrow S_{1}^{\mathbb{P} T_{1}} \rrbracket={ }_{d f} \llbracket\left(S_{0} \wedge S_{1}\right) \uparrow T_{1} \rrbracket
$$

Arguably our version is all that is required, since the standard definition permits $T_{1}$ to introduce new components and this is, perhaps, slightly odd for a restriction operation. In order to work with the standard definition we could use the general hiding operation over schema types in a manner similar to that employed below in Section 2.6, but we shall not give the details here.

The rules are then just a special case of those for conjunction. Using rules $\left(Z_{\wedge}\right)$ and $\left(Z_{\mathbb{P} \uparrow}\right)$ we obtain the type rule:

$$
\frac{\Gamma \triangleright S_{0}: \mathbb{P} T_{0} \quad \Gamma \triangleright S_{1}: \mathbb{P} T_{1} \quad T_{1} \preceq T_{0}}{\Gamma \triangleright S_{0} \uparrow S_{1}: \mathbb{P} T_{1}}
$$

The introduction and elimination rules are then as follows:

$$
\frac{\Gamma \vdash t \in S_{0} \quad \Gamma \vdash t \uparrow T_{1} \in S_{1} \quad T_{1} \preceq T_{0}}{\Gamma \vdash t \uparrow T_{1} \in S_{0} \uparrow S_{1}}\left(S_{\uparrow}^{+}\right)
$$

This follows by rules $\left(S_{\wedge}^{+}\right)$and $\left(\epsilon_{\uparrow}\right)$, noting that $T_{1}=T_{1} \vee T_{1}$ and $T_{0}=T_{0} \vee T_{1}$.

$$
\frac{\Gamma \vdash t \in S_{0} \uparrow S_{1} \quad \Gamma^{-}, y: T_{0} ; \Gamma^{+}, y \in S_{0}, y \uparrow T_{1}=t \vdash P}{\Gamma \vdash P}\left(S_{\digamma_{0}}^{-}\right) \text {, for fresh } y
$$

$$
\frac{\Gamma \vdash t \in S_{0} \uparrow S_{1}}{\Gamma \vdash t \in S_{1}}\left(S_{\Upsilon_{\mathrm{I}}}^{-}\right)
$$

These follow directly from the rules $\left(S_{\wedge_{0}}^{+}\right),\left(S_{\Lambda_{\mathrm{I}}}\right)$ and $\left(\epsilon_{\uparrow}^{-}\right)$noting that $t^{T}=t \uparrow T$.

The substitution rule is:

$$
\frac{\Gamma \vdash S_{0}^{\mathbb{P} T_{0}}=S_{2} \quad \Gamma \vdash S_{1}^{\mathbb{P} T_{1}}=S_{3} \quad T_{1} \preceq T_{0}}{\Gamma \vdash S_{0}\left\lceil S_{1}=S_{2} \uparrow S_{3}\right.}
$$

\subsection{Schema level hiding}

Our basic hiding operation takes a single label (with its type) as an argument and, as we explained earlier, does duty for what, in other accounts, is a simple form of 
schema existential quantification. In those accounts one also finds quantification over schemas in the category of schema expressions, for example [Spi92] (p. 76). We should provide, within our framework, a form of schema level hiding to correspond to this. This kind of operation has turned out to be of considerable value in the structuring of $\mathrm{Z}$ specifications. [WoD96] provides some excellent examples of operation promotion (ibid. chapter 13, see e.g. p. 187) which utilise this operation in order to promote an operation on a simple state to an operator on a global state of which it is a component. We shall return to this application in Section 4.

The definition is quite simple. In view of our earlier development we can define this easily using schema conjunction and restriction. Let $T_{1} \preceq T_{0}$ :

$$
\llbracket S_{0}{ }^{\mathbb{P} T_{0}} \backslash S_{1}{ }^{\mathbb{P} T_{1}} \rrbracket==_{d f} \llbracket\left(S_{0} \wedge S_{1}\right) \uparrow\left(T_{0} \backslash T_{1}\right) \rrbracket
$$

Using rules $\left(Z_{\wedge}\right),\left(Z_{\mathbb{P} \uparrow}\right)$, together with the fact that $T_{1} \leq T_{0}$, we obtain the following type rule:

$$
\frac{\Gamma \triangleright S_{0}: \mathbb{P} T_{0} \quad \Gamma \triangleright S_{1}: \mathbb{P} T_{1} \quad T_{1} \preceq T_{0}}{\Gamma \triangleright S_{0} \backslash S_{1}: \mathbb{P}\left(T_{0} \backslash T_{1}\right)}
$$

The introduction rule is calculated using rules $\left(S_{\uparrow}^{+}\right),\left(S_{\wedge}^{+}\right)$and the fact that $T_{0} \backslash T_{1} \preceq T_{0}$.

$$
\frac{\Gamma \vdash t \in S_{0} \quad \Gamma \vdash t \uparrow T_{1} \in S_{1} \quad T_{1} \preceq T_{0}}{\Gamma \vdash t \uparrow\left(T_{0} \backslash T_{1}\right) \in S_{0} \backslash S_{1}}
$$

The elimination rule is obtained using rule $\left(\epsilon_{\uparrow}^{-}\right)$. Let $x$ be fresh and $\Gamma^{\prime}$ be $\Gamma^{-}, x: T_{0} ; \Gamma^{+}, x \in S_{0}, x \uparrow T_{1} \in S_{1}, x \uparrow\left(T_{0} \backslash T_{1}\right)=t:$

$$
\frac{\Gamma \vdash t \in S_{0}^{\mathbb{P} T_{0}} \backslash S_{1}^{\mathbb{P} T_{1}} \quad \Gamma^{\prime} \vdash P \quad T_{1} \preceq T_{0}}{\Gamma \vdash P}
$$

There is a useful equational rule for schema level hiding. This may be compared with the syntactic characterisation of (a simpler form of) schema existential quantification which is given in [WoD96] (p. 178). Let $\alpha D_{1}=\left\{\cdots l_{i} \cdots\right\}$ and $\sigma=\left[\cdots l_{i} \cdots / \cdots z_{i} \cdots\right]$ where the $z_{i}$ are fresh variables.

$$
\frac{\Gamma^{-} \triangleright\left[D_{0} \mid P_{0}\right]: \mathbb{P} T_{0} \quad \Gamma^{-} \triangleright\left[D_{1} \mid P_{1}\right]: \mathbb{P} T_{1} \quad\left[D_{1}\right] \preceq\left[D_{0}\right]}{\Gamma \vdash\left[D_{0} \mid P_{0}\right] \backslash\left[D_{1} \mid P_{1}\right]=\left[\left[D_{0}\right] \backslash\left[D_{1}\right] \mid \exists D_{1} \sigma \bullet\left(P_{0} \wedge P_{1}\right) \sigma\right]}
$$

The substitution rule is:

$$
\frac{\Gamma \vdash S_{0}^{\mathbb{P} T_{0}}=S_{2} \quad \Gamma \vdash S_{1}^{\mathbb{P} T_{1}}=S_{3} \quad T_{1} \preceq T_{0}}{\Gamma \vdash S_{0} \backslash S_{1}=S_{2} \backslash S_{3}}
$$

\subsection{Definite description}

Although definite descriptions nominally appear in $\mathrm{Z}$ as terms it is clear, from those sources which provide a logic for $Z$, that these terms must be understood to appear syncategorematically: the rules in [WoD96] and [Nic95] are expressed in terms of equality propositions, which we will write:

$$
\mu x \in T \bullet P=t
$$


Further evidence for this being the correct approach comes from [Spi88] in which the author remarks that (the meta-theory of) $\mathrm{Z}$ can be modelled within $\mathrm{ZF}$ set-theory without the axiom of choice. The salient point being that ZF with an explicit operator for definite descriptions (such as Hilbert's epsilon or Russell's iota) would imply the axiom of choice (see e.g. [Lei69]).

The characteristic formula for definite descriptions, $\mu x \in C \bullet P=t$, is translated into $\mathrm{Z}$ by means of:

$$
\mu x \in C \bullet P=t=_{d f}\left(\exists_{1} x \in C \bullet P\right) \wedge P[x / t]
$$

and then all references to such terms may be removed by the following contextual definition into $Z$. Let $z$ be a fresh variable:

$$
P_{0}\left[z / \mu x \in C \bullet P_{1}\right]=_{d f} \exists z \in C \bullet \mu x \in C \bullet P_{1}=z \wedge P_{0}
$$

Given the definition we easily obtain the following derived rules of typing and inference:

$$
\begin{array}{cc}
\frac{C: \mathbb{P} T \quad z: T \triangleright P \text { prop }}{\mu z \in C \bullet P: T}\left(Z_{\mu}\right) \quad \frac{\exists_{1} z \in C \bullet P \quad t \in C \quad P[z / t]}{\mu z \in C \bullet P=t}\left(\mu^{+}\right) \\
\frac{\mu z \in C \bullet P=t}{t \in C}\left(\mu_{\circ}^{-}\right) \quad \frac{\mu z \in C \bullet P=t}{P[z / t]}\left(\mu_{\mathrm{I}}^{-}\right)
\end{array}
$$

With definite description in place we have immediately a means for dealing with partial application. Given $f \in T_{0} \rightarrow T_{1}$ and $x \in T_{0}$ we can set:

$$
f(x)=_{d f} \mu y \in T_{1} \bullet(x, y) \in f
$$

and then rules for partial application follow from those above for definite description. This approach is unlikely to satisfy many, since the issue of reasoning with partial terms is a research topic which extends far beyond the study of $\mathrm{Z}$ (see e.g. [Jon95]) and within which controversy is the watchword. Indeed, the reliance upon the contextual (syntactically based) introduction of definite description does not satisfy us. Investigating this topic further is, however, not the thrust of our work here.

\subsection{Conditional terms}

With definite descriptions in hand we have a method for interpreting the conditional terms often employed in example $\mathrm{Z}$ specifications.

$$
\text { 【if } P \text { then } t_{0}^{T} \text { else } t_{1}^{T} \rrbracket={ }_{d f} \llbracket \mu x \in T \bullet\left(P \Rightarrow x=t_{0}\right) \wedge\left(\neg P \Rightarrow x=t_{1}\right) \rrbracket
$$

The following type assignment rule is then derivable using rules $\left(Z_{\mu}\right),\left(Z_{\wedge}\right),\left(Z_{\Rightarrow}\right)$, $\left(Z_{=}\right),\left(Z_{\neg}\right)$ and lemmas 4.3(i) and 4.6 from [HeR99]:

$$
\frac{\Gamma \triangleright P \text { prop } \Gamma \triangleright t_{0}: T \quad \Gamma \triangleright t_{1}: T}{\Gamma \triangleright \text { if } P \text { then } t_{0} \text { else } t_{1}: T}
$$

The following introduction rule can be derived, using the law of the excluded middle for the proposition $P$ :

$$
\frac{P \vdash t_{0}=t_{2} \neg P \vdash t_{1}=t_{2}}{\left(\text { if } P \text { then } t_{0} \text { else } t_{1}\right)=t_{2}}
$$




\subsection{Generic schemas}

A generic schema is parameterised over one or more types. These are very easily accommodated within our regime. We will permit an extension of our language of types to include type variables:

$$
T::=\cdots \mid X
$$

Then we may introduce a new category of generic schemas:

$$
G S::=S[X]
$$

Finally we extend the language of schema expressions to include instantiated generic schemas:

$$
S::=\cdots \mid S[X:=T]
$$

Such instantiated schemas are interpreted into $\mathrm{Z}$ by means of:

$$
\llbracket S[X:=T] \rrbracket=_{d f} \llbracket S \rrbracket[X / T]
$$

The following rules are then immediate:

$$
\frac{P[\alpha[D] / t . \alpha[D]] \quad t \in[D][X / T]}{t \in[D \mid P][X:=T]} \quad \frac{t \in[D \mid P][X:=T]}{P[\alpha[D] / t . \alpha[D]]} \quad \frac{t \in[D \mid P][X:=T]}{t \in[D][X / T]}
$$

\subsection{Alternative forms of quantification}

There are several different forms of quantification which are adopted in the literature on Z. We shall, in this short section, only attempt to develop those alternatives presented in one of the normative sources: [Spi92]. The basic form of existential quantification ([Spi92] p. 70) is:

\section{$\exists S \bullet P$}

We shall interpret this by means of the following definitional extension, where $z$ is fresh:

$$
\llbracket \exists S \bullet P \rrbracket=_{d f} \llbracket \exists z \in S \bullet P[\alpha S / z . \alpha S] \rrbracket
$$

As a consequence we would then induce the following rules:

$$
\frac{S: \mathbb{P} T \quad z: T \triangleright P[\alpha S / z . \alpha S] \text { prop }}{\exists S \bullet P \text { prop }} \quad \frac{P[\alpha S / t . \alpha S] t \in S}{\exists S \bullet P}
$$

Let $y$ be a fresh variable in the following rule:

$$
\frac{\Gamma \vdash \exists S \bullet P_{0} \quad \Gamma^{-} \triangleright S: \mathbb{P} T \quad \Gamma^{-}, y: T ; \Gamma^{+}, y \in S, P_{0}[\alpha S / y . \alpha S] \vdash P_{1}}{P_{1}}
$$

The basic forms for $\lambda$-expressions and definite descriptions in [Spi92] (p. 58) are:

$$
\lambda S \bullet t
$$

and:

$$
\mu S \bullet P
$$

We shall omit the translation of these (and their induced rules) since they follow the pattern we have just presented for the existential quantifier and are easily 
calculated by analogy. The primitive form for set comprehension in [Spi92] ( $\mathrm{p}$. 57) is:

$\{S \bullet t\}$

We interpret this by means of the following definition. Let $z$ be a fresh variable:

$$
\llbracket\left\{S \bullet t^{T}\right\} \rrbracket=_{d f} \llbracket\{z \in T \mid \exists S \bullet z=t\} \rrbracket
$$

We then obtain the following type assignment rule:

$$
\frac{S: \mathbb{P} T_{1} \quad z: T_{1} \triangleright t[\alpha S / z . \alpha S]: T_{0}}{\{S \bullet t\}: \mathbb{P} T_{0}}
$$

The the introduction rule for this form of set comprehension is:

$$
\frac{\Gamma^{-} \triangleright S: \mathbb{P} T_{1} \quad \Gamma \vdash t_{2} \in S \quad \Gamma \vdash t_{0}=t_{1}\left[\alpha S / t_{2} . \alpha S\right]}{\Gamma \vdash t_{0} \in\left\{S \bullet t_{1}\right\}}
$$

Let $y$ be a fresh variable in the following elimination rule:

$$
\frac{\Gamma \vdash t \in\left\{S \bullet t_{1}\right\} \quad \Gamma^{-} \triangleright S: \mathbb{P} T_{1} \quad \Gamma^{-}, y: T_{1} ; \Gamma^{+}, y \in S, t=t_{1}[\alpha S / y . \alpha S] \vdash P_{1}}{P_{1}}
$$

\subsection{The mathematical toolkit}

We have said nothing about functions, sequences, bags etc. and the notation and operations which correspond to them. The reason for this is that these remaining features of $\mathrm{Z}$ are simple definitional extensions. As an example recall the standard definition of sequences. In our notation this would be:

$$
\text { seq } T={ }_{d f}\{f \in \mathbb{N} \leftrightarrow T \mid \operatorname{dom} f=1 . . \# f\}
$$

which itself requires the development of finite partial functions; which in turn is defined (e.g. [Spi92] (p. 112)) in terms of partial functions etc. The corresponding display form $\langle\cdots\rangle$ is of type $\mathbb{P}(\mathbb{N} \times T)$ and so is interpreted in terms of the display form for tuples. These details, and all the others, are completely covered in e.g. [Spi92].

\subsection{Organisation of specifications}

$\mathrm{Z}$ provides mechanisms for the overall organisation of specifications into paragraphs and sections. Where rules for these have been provided they have turned out to be among the most complex required, and are clearly the result of much ingenuity. In [Toy97] (p. 43) there are typechecking rules for sections and paragraphs which are enormously complex and which require extremely baroque side-conditions. At the very least these rules establish a useful basis for further work. The complications occurring in the rule for sections arise because each component induces a context which subsequent components inherit. It remains to be seen to what extent these complications are tamed by treating schema components as constants rather than variables. It is not even clear that these larger scale entities are best treated explicitly within the object logic itself. Their function is organisational rather than logical and the rules are more akin to side-condition 
calculations (ensuring proper scoping and so on) than to deduction. This is clearly an important topic, especially in the context of the development of support tools, and perhaps best left to those engaged in that research area.

\section{Priming and the $\theta$ operator}

There are two operations, very commonly utilised in $\mathrm{Z}$ specifications, which we have, until now, avoided entirely. In this section we shall explain and demonstrate the mathematical problems which they, jointly, cause. Following this we will describe an alternative means by which the services they are meant to provide can be presented, with the added advantage that the formalisation is relatively simple, comprehensible and, consequently, usable.

There are two competing perspectives on schemas in Z, as they are currently understood, which are mutually incompatible. The older view is that a schema is a "piece of mathematical text" ([WoD96] p. 148) or the description of a state (e.g. [She95] p. 202). The more recent, dating roughly from the time when schemas became routinely used as sets and the $\theta$ operator was introduced, is that a schema is a "set of bindings" [WoD96] (p. 156) or "collection of possible values" [She95] (p. 199). The most striking example of this appears in [Di194] (pp. 46-7), where, within two paragraphs, the author gives both accounts of schemas ${ }^{2}$.

“... Schemas are used $\cdots$ to make precise what the state space of a given specification is. The state space is defined by means of a state schema." ([Dil94], Section 4.3.4, p. 46. Our emphasis.) "PhoneDB is the name of a schema which represents a before state. Decorating the name with a prime, for example PhoneDB', represents the after state." ([Di194], Section 4.3.4, p. 47. Our emphasis.)

The older perspective accounts for the use of schema priming: if $S$ is a schema representing the before state (singular), then $S^{\prime}$ represents the after state. The notion of the $\Delta$-schema is paradigmatic of this view. It is somewhat surprising to discover that the $\Xi$-schema is paradigmatic of the alternative perspective. To see this we must first see what goes wrong when we attempt understand such schemas from the older perspective. Consider the schema $\Xi S={ }_{d f}\left[\Delta S \mid \theta S=\theta S^{\prime}\right]$. It is very well-known that in the context of the definition $T={ }_{d f} S^{\prime}$ the schema $[S ; T \mid \theta S=\theta T]$ is not even well-typed a fortiori not equal to $\Xi S$. But instead of tracing this unfortunate observation back to the root cause (the clash of perspectives we have introduced) a range of mathematically unpleasant manoeuvres have taken place in order to accommodate the situation. For example, in order to prevent Leibnitz's principle from failing one must ensure that the expression $\theta S^{\prime}$ is not the application of $\theta$ to the schema $S^{\prime}$ which ensures that the substitution is invalid. But this may not be enough: generally, $\theta$ may only be applied to schema names. This has the effect of making $\theta$ a non-extensional

\footnotetext{
2 We realise, of course, that the range of text books need not necessarily reflect our best understanding of the subject, for which we might turn to [Spi88], [Spi92] and some sections of [Nic95]. The textbooks however fulfill a very different function here: they have been written over a substantial period of time, thus providing a historical perspective on $\mathrm{Z}$ and the evolution of its concepts. Additionally, they are an excellent repository of $Z$ in practice. The practices and perspectives of the various textbooks constitutes an accurate reflection and record of the use of $Z$ within the community of its practitioners over time. The more technical references, like our own, are to a greater or lesser degree attempts to normalise or to account for that practice. What we are highlighting here, in this section, is not an inconsistency which appears only within or between some informal accounts of Z, but a genuine evolution in the community's self-understanding of $\mathrm{Z}$.
} 
operation. These devices may prevent ambiguity and avoid the incoherence of a failure of Leibnitz's principle, but they do so by technical means which are complex and unwieldy, making formalisation extremely difficult and, even if achieved, of limited value. The problem is, rather, that the type of $S^{\prime}$ is not the type required: we need the type of $S$ here. Indeed we do: the $\Xi$-schema is intended to link the initial state and the final state and these, under the second perspective, are both elements of $S$. It appears that the $\theta$ operation is inextricably linked with this second perspective. But from this viewpoint the $\Delta$-schema is incomprehensible, for it appears to suggest that operations change specifications of states (state spaces) rather than states. The solution to all this must begin by reconciling these pre-theoretic contradictions.

The older perspective, that schemas are states, is highly syntactic and it is linked with interpretations of the notation which are essentially based on macroexpansion. These have no, or very limited, mathematical properties. Moreover, this view is incompatible with almost all of the innovative work on $\mathrm{Z}$ which has taken place more recently, much of which has been introduced as a result of applying $\mathrm{Z}$ in practice. In particular, the greater role for schemas, as firstclass entities, presupposes that they represent specifications of collections and not specifications of individuals. From this perspective it is easy to render the $\Xi$-schema by means of $\left[z, z^{\prime} \in S \mid z=z^{\prime}\right]$ for some suitable choice of labels $z$ and $z^{\prime}$. Note that it is now quite clear that $S$ describes the set of states over which the operation computes, and the before and after states both conform to that specification. As a result the type of the equality is preserved naturally, without resort to dubious technical tricks. The $\Delta$-schema is now best thought of as a declaration and not as a schema at all: $z, z^{\prime} \in S$.

So far as the $\theta$ operation is concerned, we have not needed to employ it in the definition of the $\Xi$-schema because, instead of including a schema, we have introduced a declaration over the schema as a set. But this approach can be taken whenever the $\theta$ operation is normally required. It is a natural corollary of adding schemas as sets to $\mathrm{Z}$ in the systematic fashion we are advocating: the operation $\theta$ has no role to play.

\subsection{Latent declarations}

We have argued that we should remove the concepts of schema priming and the $\theta$ operator on both conceptual and mathematical grounds. We must then investigate whether or not the language remains expressive enough for its purposes. Certainly there is a change of style. Adapting existing $Z$ specifications to our revised framework requires some care: when the $\theta$ operator is useful in standard $Z$ we would introduce a declaration of schema type, where, most often, the standard $\mathrm{Z}$ would invoke a schema inclusion.

This approach, on its own, would require more explicit use of binding projection in specifications written in our system. Compare, for example, the following in standard Z ([WoD96], p. 175) and then our revised language.

$$
\text { BoxOffice }=_{d f}[\text { seating }: \mathbb{P} \text { Seat }, \text { sold }: \text { Seat } \rightarrow \text { Customer } \mid \text { dom sold } \subseteq \text { seating }]
$$




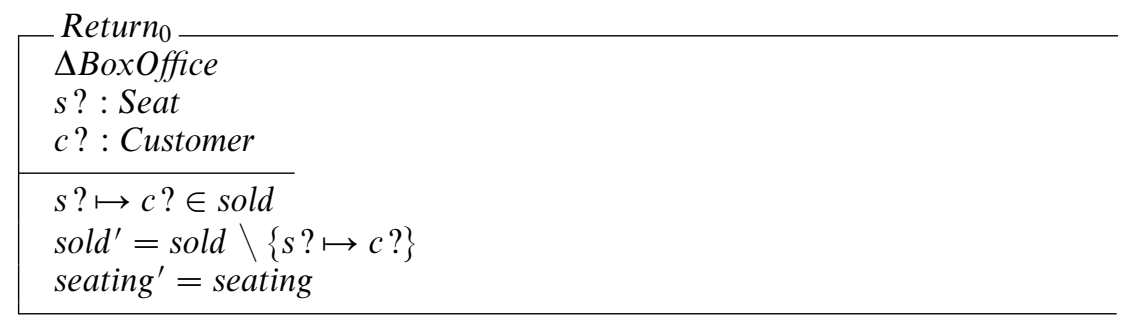

BoxOffice $=_{d f}[$ seating $\in \mathbb{P}$ Seat, sold $\in$ Seat $\rightarrow$ Customer $\mid$ dom sold $\subseteq$ seating $]$

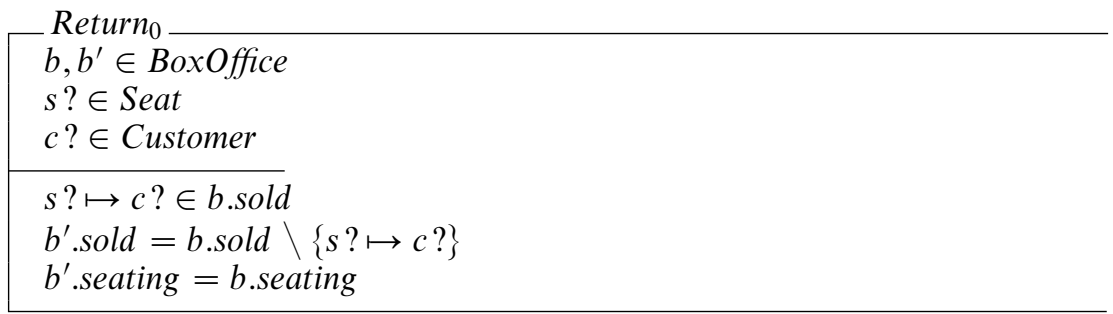

The notational burden is rather similar to that one can encounter in programs which manipulate structured data. In Pascal, for example, one has the "with" idiom to aid presentation. A generalisation of this seems called for here.

We shall permit, as prime declarations, a new form which we will call latent declarations. These are written:

$$
(l \xi \in) S
$$

where $\xi$ is a, possibly absent, diacritical mark (prime, subscript etc.). Notice that we restrict the use of this idiom to schemas only: its purpose is to ameliorate the inexpressivity of our revision of $\mathrm{Z}$ which accrues because of the occasional replacement of schema inclusion by a declaration, and there is nothing to be gained by making it more general than absolutely necessary.

The idea is that one may, in the context of this declaration, refer to the components of $S$ directly. On the other hand, $l \xi$ is available, if necessary, when one would conventionally require the $\theta$-operator.

We can translate such a novelty into $\mathrm{Z}$ by means of:

$$
\left[\cdots(l \xi \in) S^{\mathbb{P} T} \cdots \mid P\right]=_{d f}[\cdots l \xi \in S \cdots \mid P[\alpha T \xi / l \xi . \alpha T]]
$$

The diacritical mark $\xi$ plays a crucial role. It is perfectly possible (indeed highly likely in view of the inclusion of $\Delta$-schemas in operations) that a schema is effectively included twice in our version of Z Consequently, these marks, which in standard $\mathrm{Z}$ refer to distinct components in distinct schemas, allow us to determine to which declaration the component belongs.

In the presence of this syntactic device we can write the schema above as: 


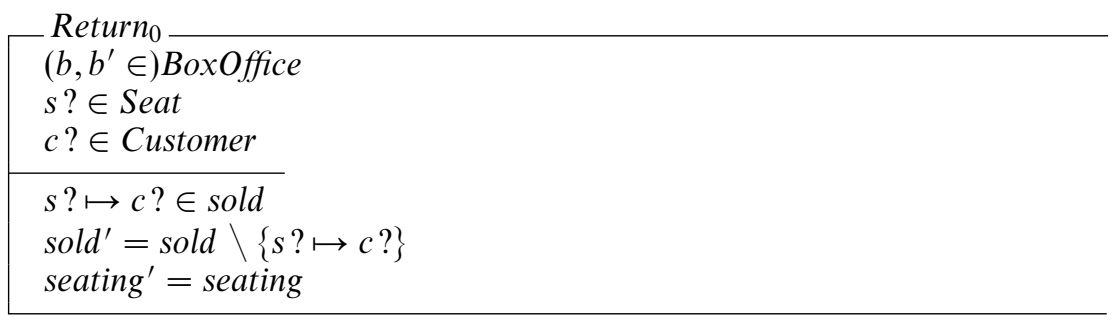

This is not significantly different from the standard presentation.

Additionally, we make use of the latently declared components at the same time as suppressing their appearance elsewhere. For example, in standard Z we might have ([WoD96] p. 193):

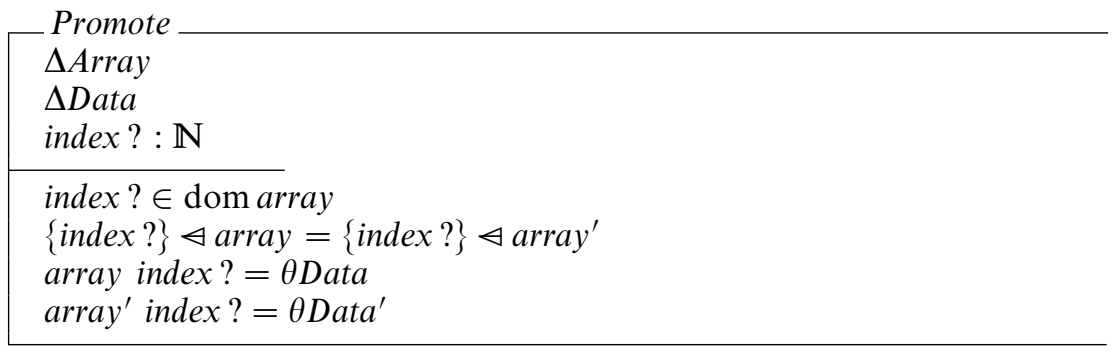

In our revised language this could now appear as:

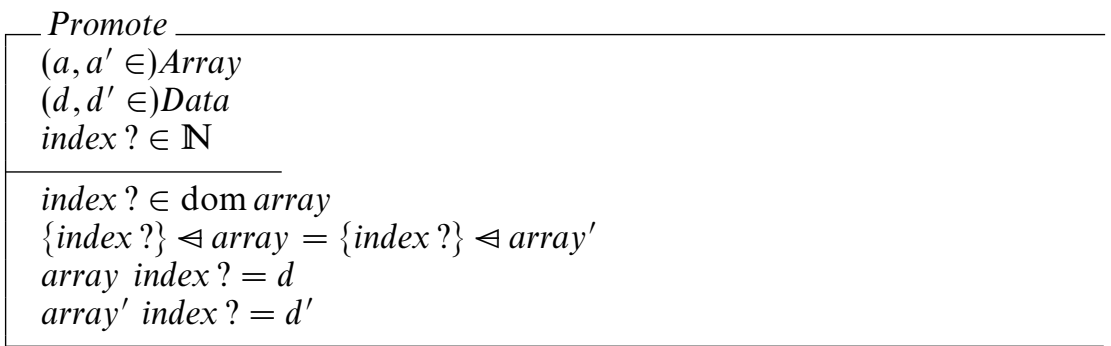

It is, perhaps, important to reinforce the point that our framework is likely to impose some differences in the style of specification. In particular, in evaluating our proposals with standard $\mathrm{Z}$ one must guard against assuming that simply transliterating existing specifications is the correct point of comparison. The following example demonstrates that one might approach a problem in quite a different way. The technique we shall illustrate is described in [Bow96] from which the example is adapted.

Example 3.1. The objective is to define a form of $\Xi$-schema which ensures that only some of the state components are invariant across a state change. Consider:

$S$ $a, b, c: \mathbb{N}$

Taking $\Delta S$ and $\Xi S$ as usual we define:

$$
\Phi(z)=_{d f} \Delta S \wedge(\Xi S \backslash[z: \mathbb{N}])
$$

Calculation reveals that $\Phi(a)=$ 


\begin{tabular}{ll}
\hline$\Delta S$ \\
\hline$b^{\prime}=b$ \\
$c^{\prime}=c$
\end{tabular}

In other words $\Phi(a)$ is the same as $\Xi S$ except that one component of $S$ (the component $a$ ) is not held invariant. Whereas we could represent this directly in our version of $Z$ we might observe that the following is possible: $\Phi[X]=_{d f}$

\begin{tabular}{l}
\hline$s, s^{\prime} \in S$ \\
\hline$s \uparrow X=s^{\prime} \uparrow X$ \\
\hline
\end{tabular}

Then we would represent the schema $\Phi(a)$ above as $\Phi[X:=[b, c: \mathbb{N}]]$.

Although we might wish to argue that this is much clearer, this is not our purpose here. The point at issue is that it is a complex matter to determine the relative expressive merits of standard $\mathrm{Z}$ and our revision, because each language determines its own natural styles. This is well worth exploring in much more detail in the future. We shall make some further comments in Section 5.

\section{Example}

We shall not try to be over ambitious and will, by no means, attempt encyclopaedic coverage of $Z$ specification techniques in this section. It will certainly remain to be seen whether or not what we have established as a revised $\mathrm{Z}$ meets the demands of practice. We would hope, at the very least, that the existence of a complete mathematical framework will encourage others to experiment.

Let us, at least, consider a reasonable example from the literature. This concerns the technique of promotion (see [WoD96] chapter 13). The example taken from this chapter (pp. 186-7) concerns the promotion of an operation over a local state to an operation over a global state. This is $Z$ at its very best: providing a general organising strategy which structures a specification. First we present the example as it stands in the book.

LocalScore

$s: \mathbb{P}$ Colour

GlobalScore

score : Players $\rightarrow$ LocalScore

AnswerLocal

$\Delta$ LocalScore

$c$ ? : Colour

$s^{\prime}=s \cup\{c ?\}$ 


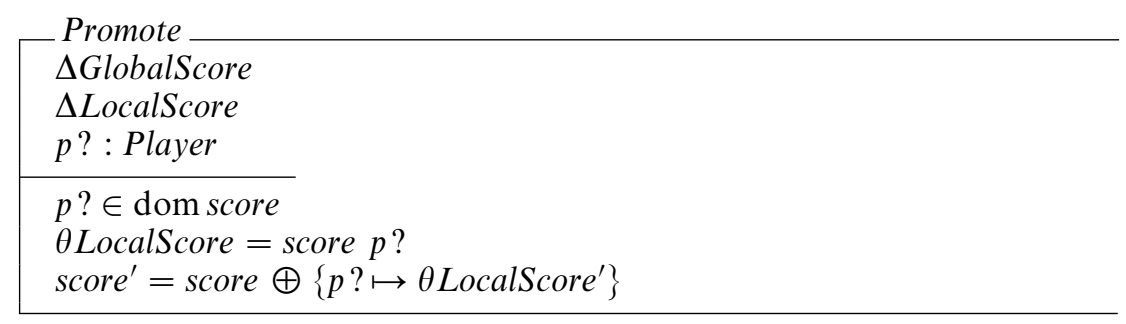

Then the specification of AnswerGlobal, the operation over the global state, is given by promoting AnswerLocal with respect to Promote:

\section{$\exists \Delta$ LocalScore $\bullet$ AnswerLocal $\wedge$ Promote}

In our presentation this would be rewritten as follows:

LocalScore

$s \in \mathbb{P}$ Colour

GlobalScore

score $\in$ Players $\rightarrow$ LocalScore

Answer Local

$\left(l, l^{\prime} \in\right)$ Localscore

$c ? \in$ Colour

$s^{\prime}=s \cup\{c ?\}$

\begin{tabular}{l} 
Promote \\
$\left(\mathrm{g}, \mathrm{g}^{\prime} \in\right)$ GlobalScore \\
$l, l^{\prime} \in$ LocalScore \\
$p ? \in$ Player \\
\hline$p ? \in$ dom score \\
$l=$ score $p ?$ \\
score ${ }^{\prime}=$ score $\oplus\left\{p ? \mapsto l^{\prime}\right\}$
\end{tabular}

Then the specification of AnswerGlobal the operation over the global state is then given by:

$$
(\text { AnswerLocal } \wedge \text { Promote }) \backslash\left[l, l^{\prime} \in \text { LocalScore }\right]
$$

What confidence can we have that the schemas we have defined are the intended interpretation? Since our operators are not defined by syntactic transformation we cannot undertake the simplification of [WoD96] p. 188 which demonstrates that $\exists \Delta$ LocalScore $\bullet$ Answer Local $\wedge$ Promote is equivalent to: 
AnswerGlobal

$\Delta$ GlobalScore

$p$ ? : Player

$c$ ? : colour

$p ? \in$ dom score

$\{p ?\} \varangle$ score $^{\prime}=\{p ?\} \varangle$ score

(score $\left.{ }^{\prime} p ?\right) . s=($ score $p ?) . s \cup\{c ?\}$

However, we have more or less the same apparatus in another guise: each of the syntactic transformations in the text-book have become instances of provable equalities in our $\mathrm{Z}$ logic. Putting together the various lemmas for the schema expressions from the technical development has established an equational logic for reasoning about schemas.

The first stage is to remove the latent declarations.

AnswerLocal
$l, l^{\prime} \in$ LocalScore
$c ? \in$ Colour
$l^{\prime} . s=l . s \cup\{c ?\}$

\begin{tabular}{|l} 
Promote \\
$g, g^{\prime} \in$ GlobalScore \\
$l, l^{\prime} \in$ LocalScore \\
$p ? \in$ Player \\
\hline$p ? \in$ dom score \\
$l=g$. score $p ?$ \\
$g^{\prime}$. score $=$ g.score $\oplus\left\{p ? \mapsto l^{\prime}\right\}$ \\
\hline
\end{tabular}

Next, since our equations always require the $D^{*}$ form of declarations, we clearly have to use the rule $\left(\epsilon^{=}\right)$on GlobalScore since its declaration part is not of the right form. GlobalScore $=_{\left(\epsilon^{-}\right)}$

$$
\begin{aligned}
& \text { GlobalScore } 0 \\
& \text { score } \in \mathbb{P}(\text { Players } \times \text { LocalScore }) \\
& \text { score } \in \text { Players } \rightarrow \text { LocalScore }
\end{aligned}
$$

We can now substitute this for GlobalScore in Promote, and then, in turn, we can equate Promote with a schema whose declaration part is in the $D^{*}$ form.

Promote $=_{\left(s u b, \in^{=}\right)}$

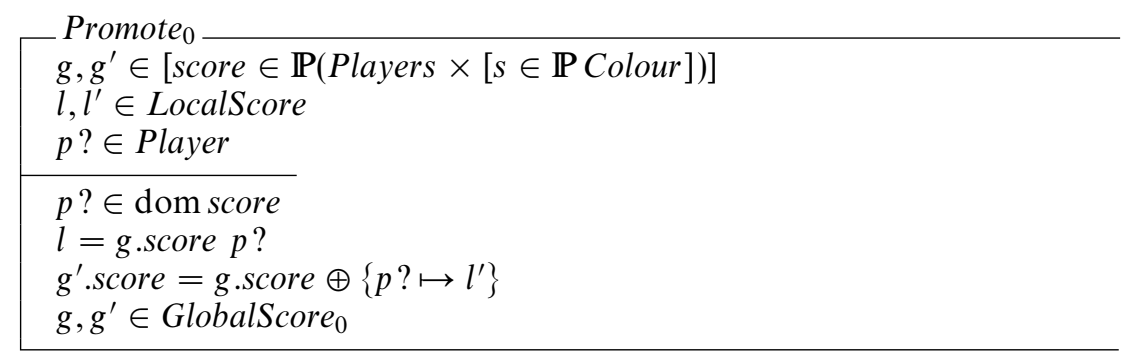


We now proceed to the conjunction:

AnswerLocal $\wedge$ Promote $=\left({ }_{(s u b)}\right.$ AnswerLocal $\wedge$ Promote ${ }_{0}=\left(\wedge^{=}\right)$

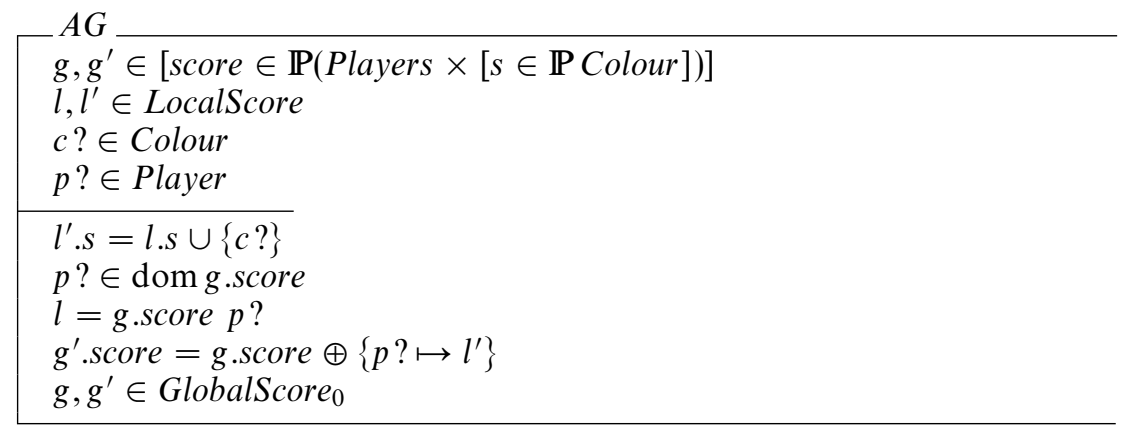

Then, by substitution, we have

AnswerLocal $\wedge$ Promote $_{0} \backslash\left[l, l^{\prime} \in\right.$ LocalScore $]={ }_{(s u b)} A G \backslash\left[l, l^{\prime} \in\right.$ LocalScore $]$ and then, by the equality rule for hiding, $A G \backslash\left[l, l^{\prime} \in\right.$ LocalScore $]=(\backslash=$

AnswerGlobal

$g, g^{\prime} \in[$ score $\in \mathbb{P}($ Players $\times[s \in \mathbb{P}$ Colour $])]$

$c ? \in$ Colour

$p ? \in$ Player

$\exists z, z^{\prime} \in$ LocalScore •

$z^{\prime} . s=z . s \cup\{c ?\} \wedge$

$p ? \in \operatorname{dom} g$.score $\wedge$

$z=g$.score $p$ ? $\wedge$

$g^{\prime}$.score $=$ g.score $\oplus\left\{p ? \mapsto z^{\prime}\right\} \wedge$

$g, g^{\prime} \in$ GlobalScore $_{0}$

Note that $z^{\prime} . s=z . s \cup\{c ?\} \Leftrightarrow z^{\prime}=\backslash s \Rightarrow z . s \cup\{c ?\} \downarrow$ is easily proved in the logic.

So the predicate part of AnswerGlobal 0 is:

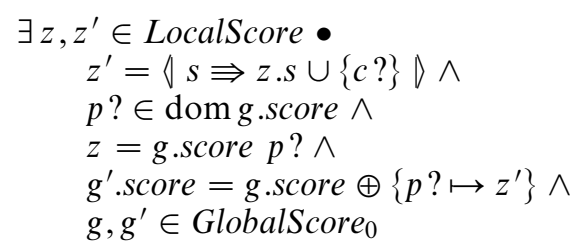

By the one-point rule, on the first equation, we have:

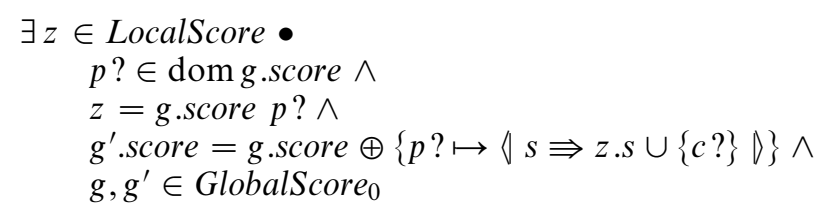

and again on the second equation gives: 


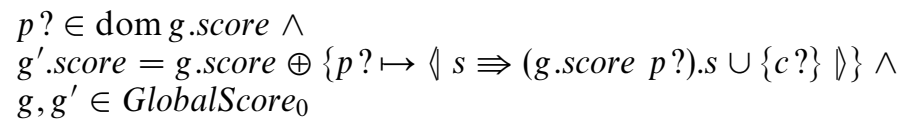

This then yields, by substitution:

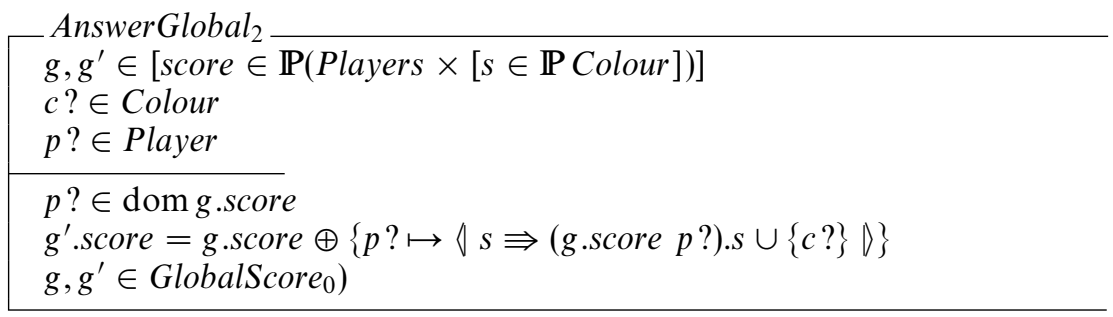

Now, using $\left(\epsilon^{=}\right)$again (and this time from right to left) we can undo the manipulations on GlobalScore we began with:

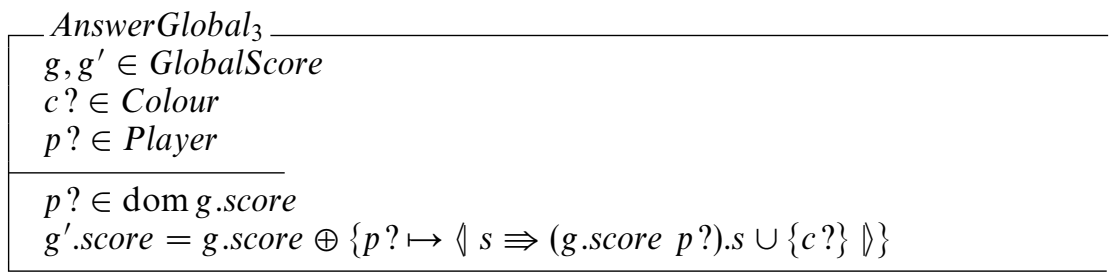

Rewriting the second equality using the same argument as [WoD96] we then have:

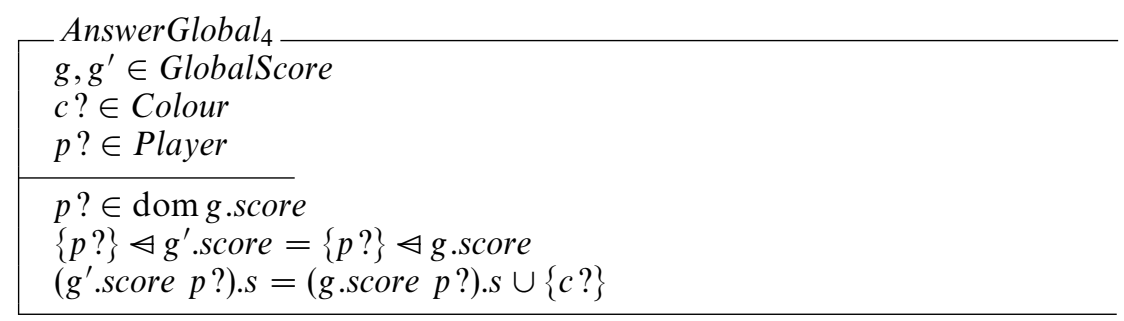

Then re-introducing latent declarations, we finally obtain:

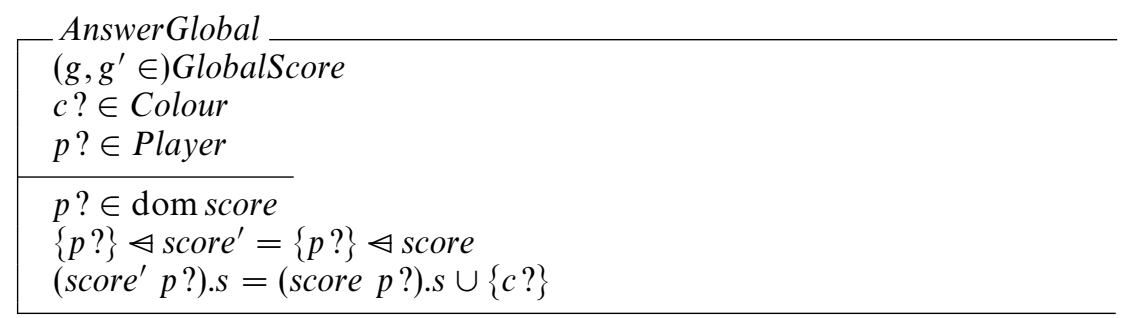

This is precisely the natural transliteration of the AnswerGlobal schema which is given in [WoD96] into our version of $\mathrm{Z}$. The equations we have developed and used are similar to the informal, purely linguistic, transformations adopted in the textbooks, and our logic explains why these work. In view of proposition 4.14 of [HeR99], deduction ensures type-correctness automatically. A logic, in fact, 
permits more to be said about specifications, and then as a consequence, more can be checked, with a gain in confidence as a major benefit.

\section{Conclusions and future work}

The purpose of this and the companion paper [HeR99] was twofold. Most crucially, we wished to provide an analysis of the language $Z$ within the context of a useful mathematical framework, thus establishing $Z$ as a specification logic. A secondary aim has been a critique of the $\mathrm{Z}$ language which has become established in the literature. These two trajectories are linked. Whilst it would have been entirely possible to outline many of the conceptual conundrums which $\mathrm{Z}$ poses in a discursive style (and it must be said that almost everything we have said is known and shared by various workers in the $Z$ research community), we have been determined to allow the mathematics to take the lead. As is very often the case, a mathematical approach does more than formalise; it additionally highlights areas of confusion and complexity. Consequently, we have used mathematical criteria to produce not only a formal account but a simple and (ultimately, we hope) usable account which retains the major benefits which $\mathrm{Z}$ offers: expressibility and scalability.

We have attempted to be reasonably comprehensive and have addressed, if in places only in outline, most of the major areas of the $\mathrm{Z}$ language. However, much remains to be done. We should like, in future publications, to develop and extend the work we have begun here on the schema calculus and, as we have mentioned, explore the organisation of specifications at the level of sections. In addition we wish to pursue program development in the context of the specification logic we have established. In particular, we are very interested in exploring other semantic foundations for $Z$ based on a constructive, intensional set theory and to compare this with the traditional model, based as it is on classical, extensional set theory.

Finally, as we acknowledge in Section 3.1, our revised framework requires a significant change in style and a significant investigation in which existing strategies are re-expressed must be undertaken. The results of such an investigation must then be used to evaluate and modify our approach. Such an interplay between theory and practice is vital. It is also not clear how the revised language interacts with existing work on program development. From our point of view this is not a concern for, as we indicated in the previous paragraph, we aim to address this topic by replacing the standard classical, extensional model with an intensional and constructive model. However, there are clearly interesting avenues to explore which utilise more conventional mechanisms. In order to investigate any of the these topics deeply, it would be very useful to use the systems provided here as the basis for a proof development tool. Work on this has already begun [Vö198], though much remains to be achieved.

\section{Acknowledgements}

We would like to thank the Department of Computer Science at the University of Waikato, New Zealand, the Centre for Discrete Mathematics and Theoretical Computer Science, New Zealand, the Royal Society of Great Britain, and the EPSRC (grant number GR/L57913) for financial assistance which has supported the development of this research. We are most grateful to Doug Goldson, Lindsay 
Groves, Ian Toyn, Ray Turner and Mark Utting for many useful discussions. We are particularly grateful to the Editor-in-Chief, Cliff Jones, for his help and encouragement in the preparation of the final version of this paper. Finally, our thanks go to one of the referees, who performed the most thorough and fruitful task of refereeing that we have ever experienced.

\section{References}

[BrM96] Brien, S. and Martin, A.: A tutorial of proof in standard Z. Technical report, Technical monograph PRG-120, University of Oxford, 1996.

[Bow96] Bowen, J.: Formal specification and documentation using Z. International Thompson Computer Press, 1996.

[Dil94] Diller, A.: Z: An introduction to formal methods ( $2^{\text {nd }}$ ed.). J. Wiley and Sons, 1994.

[HeR98] Henson, M. C. and Reeves, S.: A logic for the schema calculus. In J. P Bowen, Andreas Fett, and Michael G. Hinchey, editors, 11th Int. Conf. ZUM'98: the Z Formal Specification Notation, LNCS 1493, pages 172-191. Springer-Verlag, 1998.

[HeR99] Henson, M. C. and Reeves, S.: Revising Z: Part I - logic and semantics. Formal Aspects of Computing Journal, 11(4): 359-380, 1999.

[Jon95] Jones, C. B.: Partial functions and logics: A warning. Information Processing Letters, 54(2):65-67, 1995.

[Lei69] Leisenring, A. C.: Mathematical logic and Hilbert's $\epsilon$-symbol. McDonald Technical and Scientific, 1969.

[Mar98] Martin, A.: A revised deductive system for Z. Technical report, Technical Report TR9821, SVRC, University of Queensland, 1998.

[Nic95] Nicholls, J. (ed.): Z Notation: Version 1.2. Z Standards Panel, 1995.

[She95] Sheppard, D.: An introduction to formal specification with $Z$ and VDM. McGraw-Hill International, 1995.

[Spi88] Spivey, J. M.: Understanding Z: A specification language and its formal semantics. C.U.P., 1988.

[Spi92] Spivey, J. M.: The $Z$ notation: A reference manual. Prentice Hall, 1992.

[Toy97] Toyn, I. (ed.): Z Notation: Draft 0.8. Unpublished draft, 1997.

[Vö198] Völker, N.: Private communication, 1998.

[WoD96] Woodcock, J. and Davies, J.: Using Z: Specification, Refinement and Proof. Prentice Hall, 1996.

\section{Note added in proof}

Since this paper was accepted for publication, the draft $\mathrm{Z}$ standard [Nic95] has been superseded by the Final Committee Draft Standard [Toy99]. See the note added in proof in [HeR99] for further details.

[Toy99] Toyn, I. (ed.): Z Notation: Final Committee Draft, CD 13568.2, ftp://ftp.york.ac.uk./hise_reports/cadiz/ZSTAN/fcd.ps.gz, 1999. 